\title{
Journey into the brain: from single synapse to whole brain anatomy by correlative microscopy (Presentation Video)
}

Francesco Pavone

Francesco Pavone, "Journey into the brain: from single synapse to whole brain anatomy by correlative microscopy (Presentation Video)," Proc. SPIE 8928, Optical Techniques in Neurosurgery, Neurophotonics, and Optogenetics, 89282Y (18 March 2014); doi: 10.1117/12.2064487

SPIE. Event: SPIE BiOS, 2014, San Francisco, California, United States 


\title{
Journey into the Brain: from Single Synapse to Whole Brain Anatomy by Correlative Microscopy (Presentation Video)
}

\author{
Francesco Pavone, European Lab. for Nonlinear Spectroscopy (Italy)
}

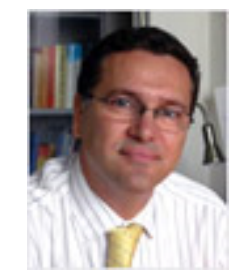

\section{BIOS 2014 Hot Topics Presentation}

The study of the brain's plasticity, or its ability to change, helps to reveal how it works. The secrets of brain activity and its control of motion are hidden in the structures, the functionality and the morphology of the physical brain, notes Francesco Pavone of the European Laboratory for Non-Linear Spectroscopy (LENS) in his presentation,"Journey into the Brain: from Single Synapse to Whole Brain Anatomy by Correlative Microscopy."

We know synapses are formed through chemical interactions and electrical connections are made, Pavone says. But in order to understand the process we must examine the brain at several different scales. A cadre of optical methods such as correlative microscopy, optical manipulation, 3D tomography, confocal light sheet microscopy, and multimodal camera imaging are used to examine the brain at highly localized regions but at the multiple scales to reveal these inner workings.

View presentation video on SPIE's Digital Library: http://dx.doi.org/10.1117/12.2064487 